\title{
Adherence to Medication, Diet and Physical Activity and the Associated Factors Amongst Patients with Type 2 Diabetes
}

\author{
Alireza Mirahmadizadeh · Haniyeh Khorshidsavar • Mozhgan Seif • \\ Mohammad Hossein Sharifi
}

Received: November 12, 2019 / Published online: January 8, 2020

(C) The Author(s) 2020

\section{ABSTRACT}

Introduction: The prevalence of type 2 diabetes mellitus (T2D) has increased considerably while the outcome of diabetic management is suboptimal. In order to promote diabetic management, associated factors for adherence to medications, diet, and physical activity (PA) need to be more clearly identified amongst patients with T2D.

Methods: This cross-sectional study was conducted on 206 men and 294 women amongst patients with diabetes who were registered in ten special diabetic clinics in Shiraz, Iran from November 2018 to April 2019. Levels of adherence to medication, Mediterranean diet (MD),

Enhanced Digital Features To view enhanced digital features for this article go to https://doi.org/10.6084/ m9.figshare.11358593.

A. Mirahmadizadeh

Non-Communicable Diseases Research Center, Shiraz University of Medical Sciences, Shiraz, Iran

H. Khorshidsavar

Shiraz University of Medical Sciences, Shiraz, Iran

M. Seif

Department of Epidemiology, School of Health, Shiraz University of Medical Sciences, Shiraz, Iran

M. H. Sharifi $(\square)$

Research Center for Traditional Medicine and History of Medicine, Shiraz University of Medical Sciences, Shiraz, Iran

e-mail: mhsharifi1350@gmail.com and physical activity were measured with validated and appropriate questionnaires.

Results: Mean age $( \pm$ SD) was $56.92 \pm 0.52$ years and 294 (58.8\%) were female. Data showed that reduced adherence to medication, MD, and PA increased glycated hemoblobin (HbA1c). Adherence to medication among low, moderate, and high levels was $27.2 \%, 59.2 \%$, and $13.6 \%$, respectively. Associated factors such as 50-64 years of age, at least 65 years of age, overweight, obese, divorced widow, smoker, and exsmoker had a significant influence on adherence to medication, $P=0.017, P=0.018, P=0.008$. $P=0.045, P=0.026, P<0.004$, and $P=0.001$, respectively. In addition, adherence to $\mathrm{MD}$ among low, moderate, and high levels was $5.4 \%$, $77.2 \%$, and $17.4 \%$, respectively. Associated factors such as 50-64 years of age, at least 65 years of age, overweight, obese, and smoker had a significant influence on adherence to $\mathrm{MD}$, $P=0.011, P=0.046, P=0.002, P<0.001$, and $P=0.032$, respectively. Furthermore, adherence to PA among low, moderate, and high levels was $21 \%, 68.6 \%$, and $10.4 \%$, respectively. Only the non-smoker factor played a significant role in PA adherence, $P=0.010$.

Conclusion: Levels of adherence to medications, diet, and PA amongst patients with T2D are not within the acceptable range. Recognized associated factors that contribute to adherence might improve diabetes management and allow one to tailor the appropriate message to improve glycemic indices. 
Keywords: Adherence; Diet; Medication; Physical activity; Type 2 diabetes

\section{Key Summary Points}

\section{Why carry out this study?}

In order to promote diabetic management, the associated factors for adherence to medications, diet, and physical activity (PA) need to be more clearly identified amongst patients with type 2 diabetes (T2D).

Although the levels of adherence to medication, diet, and physical activity have been evaluated separately in other studies in patients with T2D, few studies have simultaneously evaluated the adherence to these factors.

\section{What was learned from the study?}

Reduced adherence to medication, Mediterranean diet (MD), and PA increased HbA1c.

Adherence to medication among low, moderate, and high levels was $27.2 \%$, $59.2 \%$, and $13.6 \%$, respectively.

Associated factors such as 50-64 years of age, at least 65 years of age, overweight, obese, divorced widow, smoker, and exsmoker had a significant influence on adherence to medication, $P=0.017$, $P=0.018, P=0.008, P=0.045, P=0.026$, $P<0.004$, and $P=0.001$, respectively.

Adherence to MD among low, moderate, and high levels was 5.4\%, 77.2\%, and $17.4 \%$, respectively. Associated factors such as 50-64 years of age, at least 65 years of age, overweight, obese, and smoker had a significant influence on adherence to $\mathrm{MD}, P=0.011, P=0.046, P=0.002$, $P<0.001$, and $P=0.032$, respectively.

Adherence to PA among low, moderate, and high levels was $21 \%, 68.6 \%$, and $10.4 \%$, respectively. Only the non-smoker factor played a significant role in PA adherence, $P=0.010$.

\section{INTRODUCTION}

The prevalence of type 2 diabetes mellitus (T2D) as a chronic metabolic disorder has increased dramatically in developed and developing countries while the role optimal diabetes management strategies is not defined in detail because of the complexity of its management programs and lifestyle $[1,2]$. T2D accounts for approximately $90 \%$ of all diabetes cases worldwide. According to a projection by the World Health Organization (WHO), by 2040, 8.5\% of the world's population (642 million) will have T2D in comparison to $4.7 \%$ in 1980 [1, 3]. Between 2010 and 2030, the number of adults with diabetes will also increase by $69 \%$ in developing countries and by $20 \%$ in industrialized countries. Asia is a major region affected by the rapidly growing worldwide T2D epidemic [1]. Similar to developing countries, data revealed that Iran has had an alarming trend in T2D numbers; the prevalence of T2D amongst adults in Iran was $5.75 \%$ in 2010 and $8.7 \%$ in 2018. In general, three modifying approaches in T2D management are as follows: medication, diet, and physical activity (PA). Although the levels of adherence to medication, diet, and PA have been evaluated separately in other studies in patients with T2D, few studies have simultaneously evaluated the adherence to these factors.

Evidence showed that adherence to diet or PA in patients with diabetes is still low compared to adherence to medication. The adherence level in taking medication is crucial in the management of T2D. Medication adherence is an important determinant of therapeutic outcome such as glycemic indices and to prevent complications. According to data, adherence to medication varies from $10 \%$ to $74 \%$ in different populations $[4,5]$. Thus, this wide range might show barriers or influences of the related factors such as age, socioeconomic status, and disease duration on adherence to medication in people with diabetes.

Data shows that a Mediterranean diet (MD) is one of the best diets for diabetes patients $[6,7]$. In addition, a meta-analysis showed that higher adherence to $\mathrm{MD}$ is linked to 
cardiovascular disease improvement and reduced mortality [8]. A balanced MD emphasizes the consumption of appropriate fats, carbohydrates, and proteins that can contribute to the reduction of blood glucose and insulin resistance. Research by Esposito et al. revealed that adherence to MD can improve the glycemic indices by reducing the need for medication [9], but what they failed to do was simultaneously assess adherence to MD and medication.

There is now clear evidence that regular PA has broad health benefits, ranging from reduced cancer risk to improved chronic disease management [10, 11]. PA can also reduce blood glucose levels and increase insulin sensitivity [12]. Although PA is a key element in the management of T2D, adherence to PA is extremely poor. A meta-analysis indicted that patients with chronic diseases had $77 \%$ adherence to the prescribed PA [13]. Another study also showed that $39 \%$ of adults with diabetes reported being physically active compared to $58 \%$ of diabetes-free adults [14]. To sum up, poor PA among patients with diabetes needs to be better evaluated.

Research has to fill this knowledge gap in order to better recognize optimal diabetes management in more detail. Lifestyle modification, such as MD and PA, can affect T2D management by preventing long-term complications. Clearly, low adherence to PA, diet, and medication can gradually increase the burden of the disease [15]. This study was therefore conducted to determine the adherence to medications, MD, and PA amongst patients with T2D and to evaluate the associated variables such as sociodemographic characteristics and diabetic complications.

\section{METHODS}

This cross-sectional study was conducted on 206 male and 294 female patients with diabetes who had registered in ten special diabetic clinics in Shiraz, Iran from November 2018 until April 2019. Sociodemographic data questionnaire (gender, age, education level, marital status, etc.) and having a glucometer, smoking status, and documented diabetic complications were recorded from those individuals who agreed to participate in the study. The inclusion criteria are as follows: being over the age of 30 with a history of diabetes for 2 years or more. The exclusion criteria were autoimmune diseases, steroid consumption, and inaccessible glycated hemoglobin $(\mathrm{HbA} 1 \mathrm{c})$ records from 6 months earlier in the aforementioned special diabetic clinics.

\section{Data Sampling and Sample Size Calculation}

The purpose of this study was to determine the effect of 15 predictors on compliance. As a rule of thumb, 30 observations were required to estimate each coefficient of multiple regression. Therefore, 450 observations were the minimum sample size required. Eventually, with $10 \%$ nonresponse rate and probably missing data, 500 patients with diabetes were interviewed $[16,17]$.

\section{Ethical Approval}

This study was approved by the local Ethics Committee of Shiraz University of Medical Sciences (SUMS); IR.SUMS.REC.1397.972. In addition, written informed consent was obtained from each participant. This study was performed in accordance with the Helsinki Declaration of 1964 and its later amendments.

\section{Measurements and Questionnaires}

To obtain the adherence to medications, MD, and PA, three questionnaire were administered at the time of registration and anthropometric parameters were assessed. Patient height was measured, using a non-stretchable measuring tape to the nearest centimeter while the participants were barefoot. In addition, body weight was evaluated using SECA scales 799 (SECA, Germany) to the nearest $0.1 \mathrm{~kg}$ while the participants were wearing light clothing. Then, body mass index (BMI) (weight in kilograms divided by the square of height) was calculated. BMI between $25 \mathrm{~kg} / \mathrm{m}^{2}$ and $29.9 \mathrm{~kg} / \mathrm{m}^{2}$ is considered to be overweight and BMI greater than 
30 was considered to be obese for both men and women [18].

\section{Morisky Medication Adherence Scale (MMAS-8)}

The validated questionnaire [19] included an eight-item Morisky medication adherence scale (MMAS-8) to evaluate the level of adherence to medication. MMAS-8 includes eight yes or no questions and responses to evaluate patient adherence to medication.

\begin{tabular}{ll}
\hline \multicolumn{2}{l}{ Morisky medication adherence scale (MMAS-8) } \\
\hline Adherence rate & Questionnaire scale \\
\hline Low & $>2$ \\
Moderate & $1-2$ \\
High & 0 \\
\hline
\end{tabular}

\section{4-Point Mediterranean Diet Adherence Score (MEDAS)}

An expert researcher completed a validated and reliable 14-item modified MD adherence questionnaire $[19,20]$ in a face-to-face interview with the participants. This questionnaire was divided into three categories of MD adherence (score less than 5, low adherence; score 6-9, medium adherence; and score 10, high adherence).

\section{Physical Activity Adherence}

The International Physical Activity Questionnaire (IPAQ) is a validated and reliable instrument, designed primarily for adults (age range of 15-69 years) [21, 22]. The IPAQ face-to-face interview format was used to evaluate the PA levels of the participants. Additionally, energy consumption was calculated on the basis of the second edition of codes and metabolic equivalent (MET) values [23]. The IPAQ data were converted to MET scores (MET-min per week) for each type of activity by multiplying the number of minutes dedicated to each activity class by the specific MET score for that activity. Moreover, on the basis of the revised scoring protocol 2011, PA levels were categorized into three levels as follows: high (at least 3000 MET-min/week), moderate (at least 600 MET-minutes/week), and low (less than 600 MET-min/week) [23, 24].

\section{Statistical Analysis}

The data are expressed as mean \pm standard deviation for numeric variables and as frequency or proportion for categorical ones. Kolmogorov-Smirnov and Levene's test were used respectively to assess normality and equality of variances. Univariable and multivariable statistical analyses were used to investigate the relation among adherence to medication, MD, and PA and associated factors. Differences in associated factors and HbA1c were compared with one-way analysis of variance (ANOVA). Ordinal logistic regression models were used to assess low, moderate, and high adherence to medication protocol, MD, and PA. Crude, adjusted OR, and $0.95 \mathrm{CI}$ were estimated. For multivariate logistic regression, variables with $P$ values less than 0.2 were candidates for entrance into the model. Finally, the $P$ value of the proportional odds, which is the required assumption for ordinal logistic regression, is reported in tables. The analyses were done using the SPSS statistical software, version 22 and $P \leq 0.05$ was considered to be statistically significant.

\section{RESULTS}

Five hundred patients participated in this study and their mean age $( \pm \mathrm{SD})$ was $56.92 \pm 0.52$ years. More than half, 294 (58.8\%) were female and $412(82.4 \%)$ were married. With regard to clinical characteristics, 143 (28.6\%) participants had retinopathy, 63 (12.6\%) nephropathy, 109 (21.8\%) neuropathy, $107(21.4 \%)$ cardiovascular disease (CVD), 215 (43\%) hypertension, and 115 (23\%) diabetic foot ulcer. The majority of patients 358 (71.6\%) were under oral antidiabetic medication while $142(28.4 \%)$ were under both insulin therapy 
Table 1 Sociodemographic characteristics and HbAlc in participants

\begin{tabular}{llll}
\hline Variables & No. $\%$ & HbA1c & $P$ value \\
& Mean \pm SD & $\begin{array}{l}(T \text { test or } \\
\text { ANOVA })\end{array}$ \\
& & & ANOV)
\end{tabular}

Gender

Male

Female

206

$294 \quad 58.8 \quad 7.48 \pm 1.36$

Age group

$\begin{array}{lllll}30-49 & 134 & 26.8 & 7.07 \pm 1.08<0.001 \\ 50-64 & 229 & 45.8 & 7.73 \pm 1.51 \\ \geq 65 & 137 & 27.4 & 7.45 \pm 1.30\end{array}$

Body mass index

$\begin{array}{lllll}\text { Normal } & 112 & 22.4 & 7.22 \pm 1.56 & 0.008 \\ \text { Overweight } & 269 & 53.8 & 7.45 \pm 1.24 & \\ \text { Obese } & 119 & 22.1 & 7.78 \pm 1.43 & \end{array}$

Marital status

$$
\text { Single }
$$

Married

Others

$$
\begin{array}{rll}
19 & 3.8 & 6.86 \pm .76 \\
412 & 82.4 & 7.48 \pm 1.40 \\
69 & 13.8 & 7.63 \pm 1.33
\end{array}
$$

Education levels

\begin{tabular}{lrrrr} 
Illiterate & 102 & 20.4 & $7.64 \pm 1.14$ & 0.024 \\
$\begin{array}{l}\text { Elementary and } \\
\text { middle }\end{array}$ & 121 & 23.1 & $7.44 \pm 1.39$ & \\
$\begin{array}{l}\text { High school and } \\
\text { diploma }\end{array}$ & 193 & 38.6 & $7.58 \pm 1.62$ & \\
$\begin{array}{l}\text { Academic } \\
\text { Type of medication }\end{array}$ & 84 & 16.8 & $7.09 \pm 0.85$ & \\
$\begin{array}{l}\text { Oral } \\
\text { Insulin + OHGA }\end{array}$ & 142 & 28.4 & $7.94 \pm 1.60$ & \\
Having glucometer & & & & \\
Yes & 482 & 96.4 & $7.46 \pm 1.36$ & 0.449 \\
No & 18 & 3.6 & $7.78 \pm 1.83$ & \\
\hline
\end{tabular}

Table 1 continued

\begin{tabular}{lrlll}
\hline Variables & No. $\%$ & $\begin{array}{l}\text { HbA1c } \\
\text { Mean } \pm \text { SD }\end{array}$ & $\begin{array}{l}\boldsymbol{P} \text { value } \\
(\boldsymbol{T} \text { test or } \\
\text { ANOVA })\end{array}$ \\
\hline \multicolumn{5}{c}{$\begin{array}{l}\text { Diabetes in any } \\
\text { Yirst-degree relative }\end{array}$} \\
Yes & 235 & 47 & $7.51 \pm 1.42$ & 0.914 \\
No & 265 & 53 & $7.45 \pm 1.34$ & \\
Hookah smoker & & & & \\
Not at all & 366 & 73.2 & $7.39 \pm 1.33$ & 0.067 \\
Ex-smoker & 70 & 14 & $7.73 \pm 1.52$ & \\
Smoker & 64 & 12.8 & $7.69 \pm 1.41$ & \\
Hookah smoker & & & & \\
Not at all & 440 & 88 & $7.46 \pm 1.40$ & 0.798 \\
Ex-hookah & 26 & 6.2 & $7.48 \pm 1.22$ & \\
smoker & & & & \\
Hookah smoker & 34 & 5.8 & $7.63 \pm 1.09$ & \\
\hline
\end{tabular}

OHGA oral hypoglycemic agent

and oral antidiabetic medication. Good glycemic control (HbA1c $\leq 7 \%)$ was found in 199 $(39.8 \%)$ patients with a mean \pm SD of $7.48 \pm 0.06 \%$. Response rate was $87 \%$.

Table 1 shows sociodemographic characteristics of the participants and HbA1c. There was a significant difference between age groups, BMI, education level, and type of medication $(P=0.00, P=0.00, P=0.02, P=0.00$ respectively) in HbA1c.

Table 2 presents mean \pm SD HbA1c and compares adherence to medication protocol, $\mathrm{MD}$, and PA at three levels. Adherence to medication protocol among low, moderate, and high levels was $27.2 \%, 59.2 \%$, and $13.6 \%$, respectively. As shown, with increased adherence to medications, the concentration of HbA1c decreased. In this table, it is apparent that there were significant differences between low, moderate, and high levels of adherence to medication protocol $(P=0.00)$, according to HbA1c. In addition, we can see that adherence to MD among low, moderate, and high levels was $5.4 \%, 77.2 \%$, and $17.4 \%$, respectively. As it can be seen, with increase in MD adherence, the HbA1c concentration was reduced. In 
Table 2 Frequency of adherence to the medication protocol, Mediterranean diet, and physical activity and mean \pm SD of HbAlc

\begin{tabular}{llrrrrl}
\hline Variables & Levels of adherence & \multicolumn{2}{l}{ Frequency } & \multirow{2}{*}{ HbA1c (mean \pm SD) } & P value* \\
\cline { 3 - 4 } & & No. & \% & & \\
\hline Adherence to medication protocol & Low & 136 & 27.2 & $8.23 \pm 1.54$ & $<0.001$ \\
& Moderate & 296 & 59.2 & $7.32 \pm 1.21$ & \\
& High & 68 & 13.6 & $6.66 \pm 0.95$ & $<0.001$ \\
Adherence to Mediterranean diet & Low & 27 & 5.4 & $8.57 \pm 1.94$ & $<0.001$ \\
& Moderate & 386 & 77.2 & $7.63 \pm 1.32$ & \\
Adherence to physical activity & High & 87 & 17.4 & $6.47 \pm 0.70$ & \\
& Low & 105 & 21.0 & $9.07 \pm 1.54$ & \\
& Moderate & 343 & 68.6 & $7.12 \pm 0.92$ & \\
\hline
\end{tabular}

${ }^{*} T$ test or ANOVA

Table 3 Multiple regression to assess simultaneous effects of different adherence on HbAlc

\begin{tabular}{llllll}
\hline Effect & Coefficient & Standard error & $\boldsymbol{P}$ value & \multicolumn{2}{c}{$\mathbf{9 5 \% \text { confidence interval }}$} \\
\cline { 5 - 6 } & & & & \multicolumn{1}{c}{ Lower } & Upper \\
\hline Adherence to medication protocol & -0.30 & 0.09 & 0.001 & -0.47 & -0.13 \\
Adherence to Mediterranean diet & -0.57 & 0.12 & $<0.001$ & -0.80 & -0.34 \\
Adherence to physical activity & -1.14 & 0.10 & $<0.001$ & -1.33 & -0.95 \\
\hline
\end{tabular}

this regard, there were significant differences between low, moderate, and high levels of adherence to $\mathrm{MD}(P=0.00)$, according to HbA1c. As shown in Table 2 , there were significant differences between low, moderate, and high levels of adherence to PA $(P=0.001)$, according to HbA1c. Low, moderate, and high adherence levels were $21 \%, 68.6 \%$, and $10.4 \%$, respectively. Overall, we can see that decreased adherence to the medication protocol, MD, and PA increased HbA1c.

As all the coefficients were negative and statistically significant, it could be concluded that increasing any type of adherence would decrease HbA1c. Since all the standard errors were almost the same, the most effect size for decreasing HbA1c could be related to "Adherence to physical activity" followed by "Adherence to Mediterranean diet" and "Adherence to medication protocol". Results also showed that each level increase in adherence to physical activity, diet, and medication protocol would respectively decrease the HbA1c by almost 1 , 0.6 , and 0.3 units (Table 3 ).

Table 4 presents factors associated with low, moderate, and high adherence to medication. There was a significant difference in adherence to medication in the at least 65 years of age group $(\mathrm{OR}=0.48,95 \%$ CI $0.26-0.88)$, overweight $(\mathrm{OR}=0.53,95 \% \mathrm{CI} 0.34-0.84)$, divorced widow (OR $=0.28,95 \%$ CI 6.16-8.17), ex-smoker $(\mathrm{OR}=0.45,95 \%$ CI $0.25-0.81)$, and smoker $(\mathrm{OR}=0.24,95 \% \mathrm{CI} 0.13-0.42)$. In this respect, we can see a reduction in adherence to medication as the age increased. In addition, we can see a decrease in adherence to medication with an increase in weight. Also, singles had the 


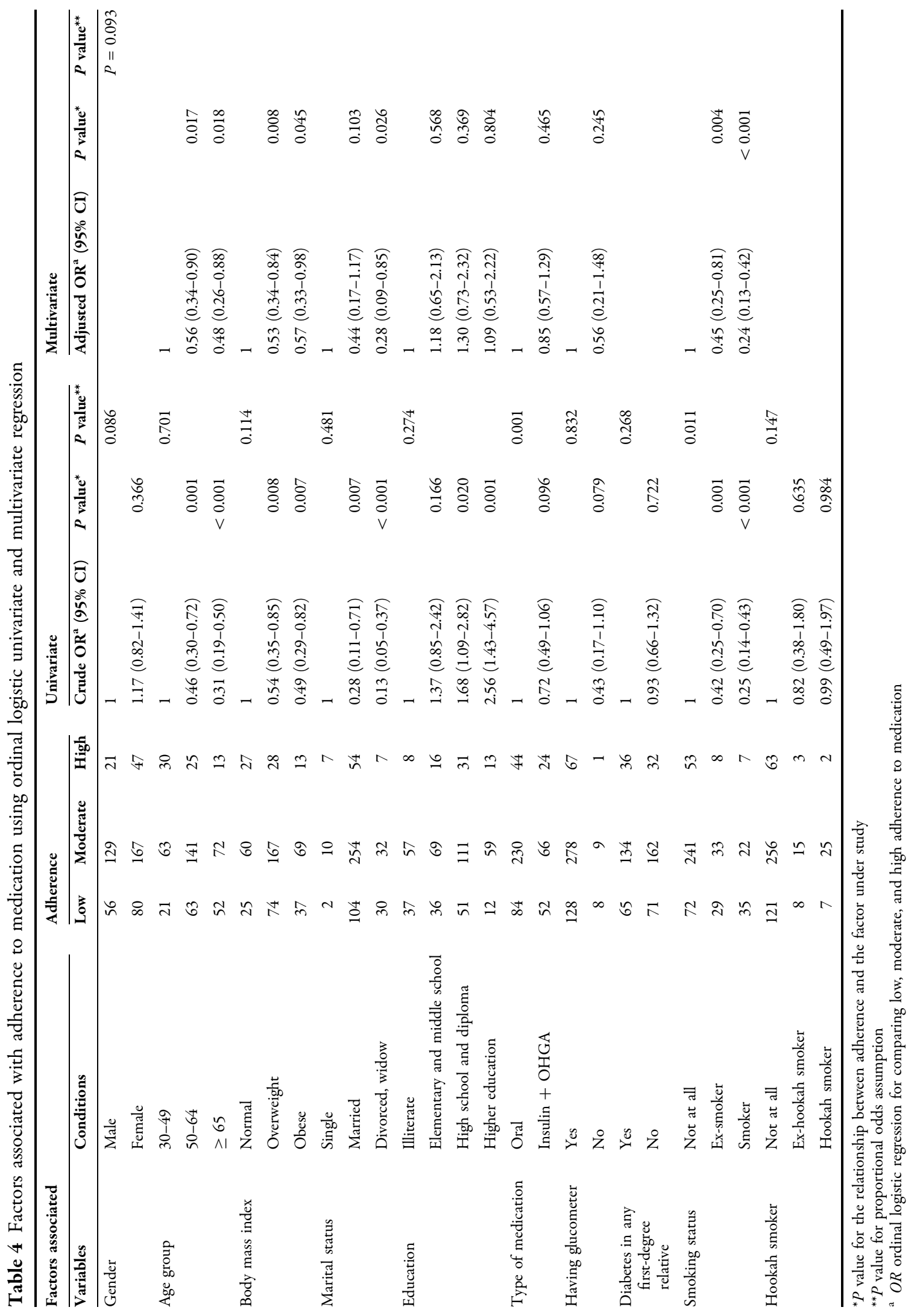


highest adherence to medication. Details are presented in Table 4 .

Table 5 presents factors associated with low, moderate, and high adherence to MD. There was a significant difference in adherence to medication in the 50-64 age group (OR $=0.49$, $95 \%$ CI $0.28-0.84)$, at least 65 age group $(\mathrm{OR}=0.47, \quad 95 \% \quad \mathrm{CI} \quad 0.23-3.71)$, overweight $(\mathrm{OR}=0.44,95 \%$ CI $0.26-0.74)$, obese $(\mathrm{OR}=$ 0.29 , 95\% CI $0.15-0.56)$, single $(\mathrm{OR}=2.84$, $95 \%$ CI 1.79-3.84), illiterate (OR $=0.29,95 \%$ CI $0.15-0.56)$, and smoker $(\mathrm{OR}=0.46,95 \% \mathrm{CI}$ $0.23-0.93)$. In this respect, we can see a reduction in adherence to MD as the age increased. Furthermore, we can see a reduction in adherence to MD with an increase in weight. In addition, the smokers had low adherence to MD. Details are presented in Table 5.

Table 6 presents factors associated with low, moderate, and high adherence to PA. There was a significant difference in adherence to PA in smoker (OR $=0.47,95 \%$ CI 0.26-0.83). Also, in terms of adherence to PA, people who had never smoked were more likely to have high adherence to PA. Details are presented in Table 6.

Table 7 presents detail information regarding the association between diabetic complications and adherence to medication protocol, MD, and PA. In this regard, adherence to medication protocol, $\mathrm{MD}$, and PA reduced the chance of retinopathy by $57 \%, 83 \%$, and $51 \%$, respectively. Moreover, adherence to medication protocol, $\mathrm{MD}$, and PA reduced the chance of neuropathy by $86 \%, 94 \%$, and $99 \%$, respectively. Also, adherence to medication protocol, $\mathrm{MD}$, and PA reduced the chance of nephropathy by $49 \%, 86 \%$, and $46 \%$, respectively. Furthermore, adherence to medication protocol, MD, and PA reduced the chance of CVD by $62 \%$, $68 \%$ and $73 \%$, respectively. Additionally, adherence to the medication protocol, MD, and PA reduced the chance of hypertension (HTN) by $99 \%, 82 \%$, and $50 \%$, respectively. In addition, adherence to the medication protocol, $\mathrm{MD}$, and PA reduced the chance of diabetic foot ulcer by $54 \%, 83 \%$, and $60 \%$, respectively.

\section{DISCUSSION}

This study was carried out to determine the association among adherence to medication, MD, and PA with sociodemographic characteristics and diabetic complications in patients with T2D. Our findings revealed that levels of adherence to MD and PA were not within the acceptable range. Overall, the data showed that reduced adherence to medication protocol, MD, and PA increased HbA1c. The results of this investigation indicated that four sociodemographic factors, namely age group (50-64 and at least 65 years of age), BMI, marital status, and smocking status, had significant influence on adherence to medication. Moreover, the associated factors, such as age groups (50-64 and at least 65 years of age), BMI, and smoking played an important role in low, moderate, and high levels of adherence to MD, whereas just the factor non-smoker played an important role in low, moderate, and high levels of adherence to PA. Adherence to medication protocol, MD, and PA also affected the reduction of diabetic complications and comorbidity.

Patient adherence to antidiabetic medication is essential in preventing the undesirable complications of diabetes and reducing the utilization of healthcare resources. According to previous studies, approximately $50 \%$ of patients with T2D were unsuccessful in attaining adequate glycemic control, and low adherence to medication was one of the primary causative factors [25]. The present study revealed that levels of low, moderate, and high adherence to medication protocol amongst patients with diabetes were $27.2 \%, 59.2 \%$, and $13.6 \%$, respectively. These results differ from those of Elsous et al., who estimated $2.5 \%, 39.5 \%$, and $58 \%$ of low, moderate, and high adherence to medication protocol $[5,20]$. These differences can be explained because adherence to medication is affected by many factors, including social and economic factors, attitudes, motivations and levels of physical/cognitive impairment of patients, severity of medical conditions, and the healthcare system in which the patient receives care. In accordance with the present result, a previous study showed that 


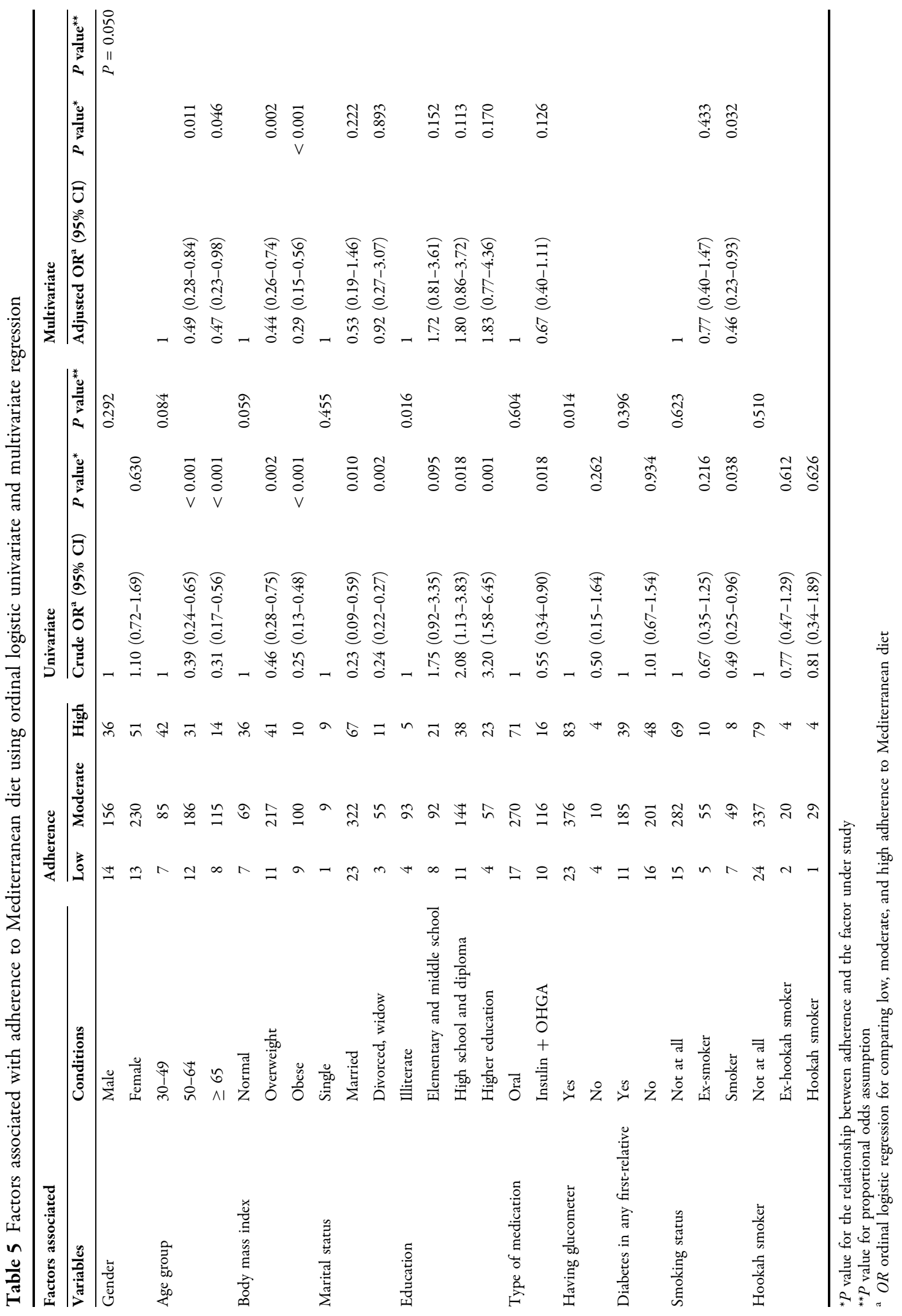




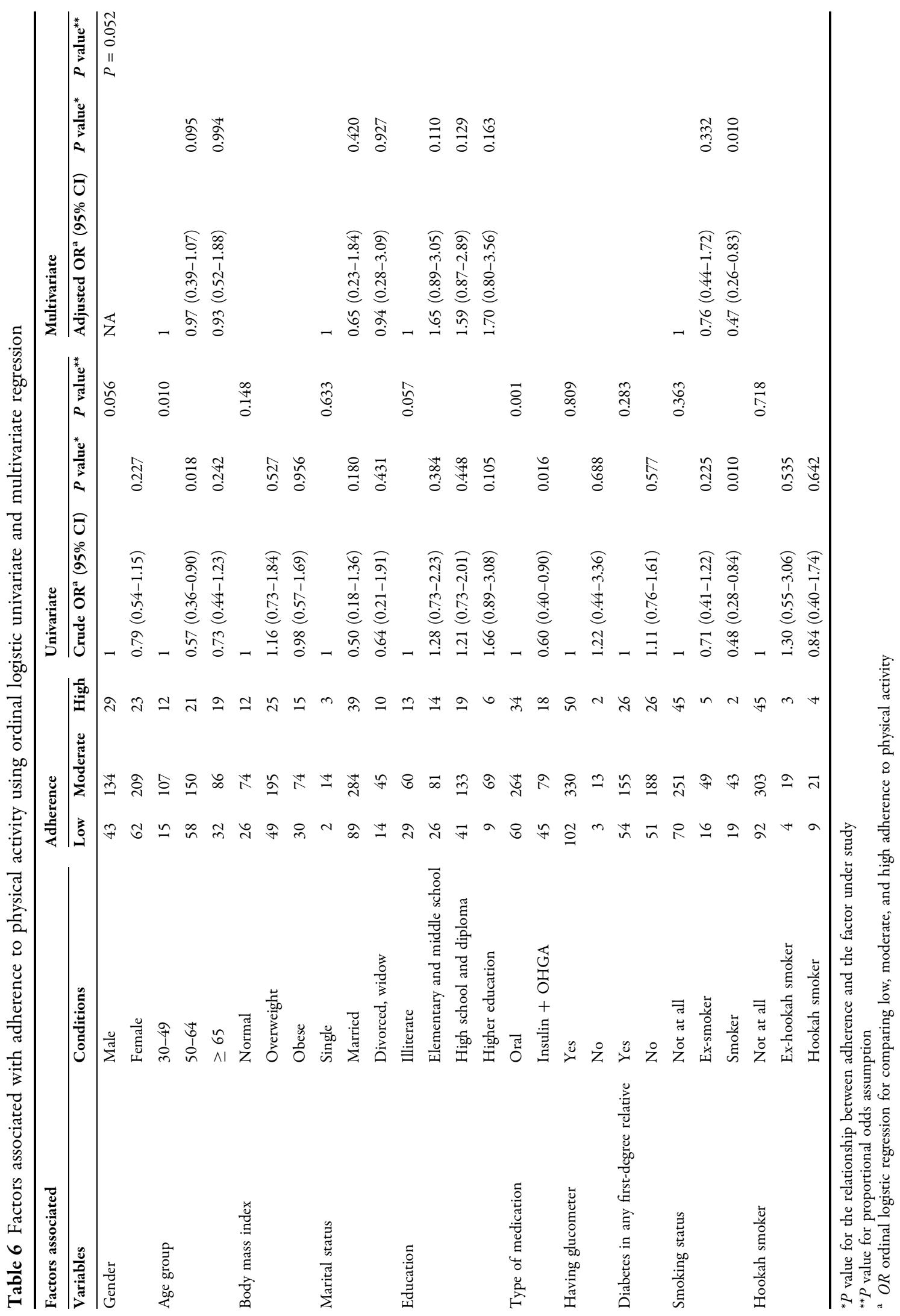




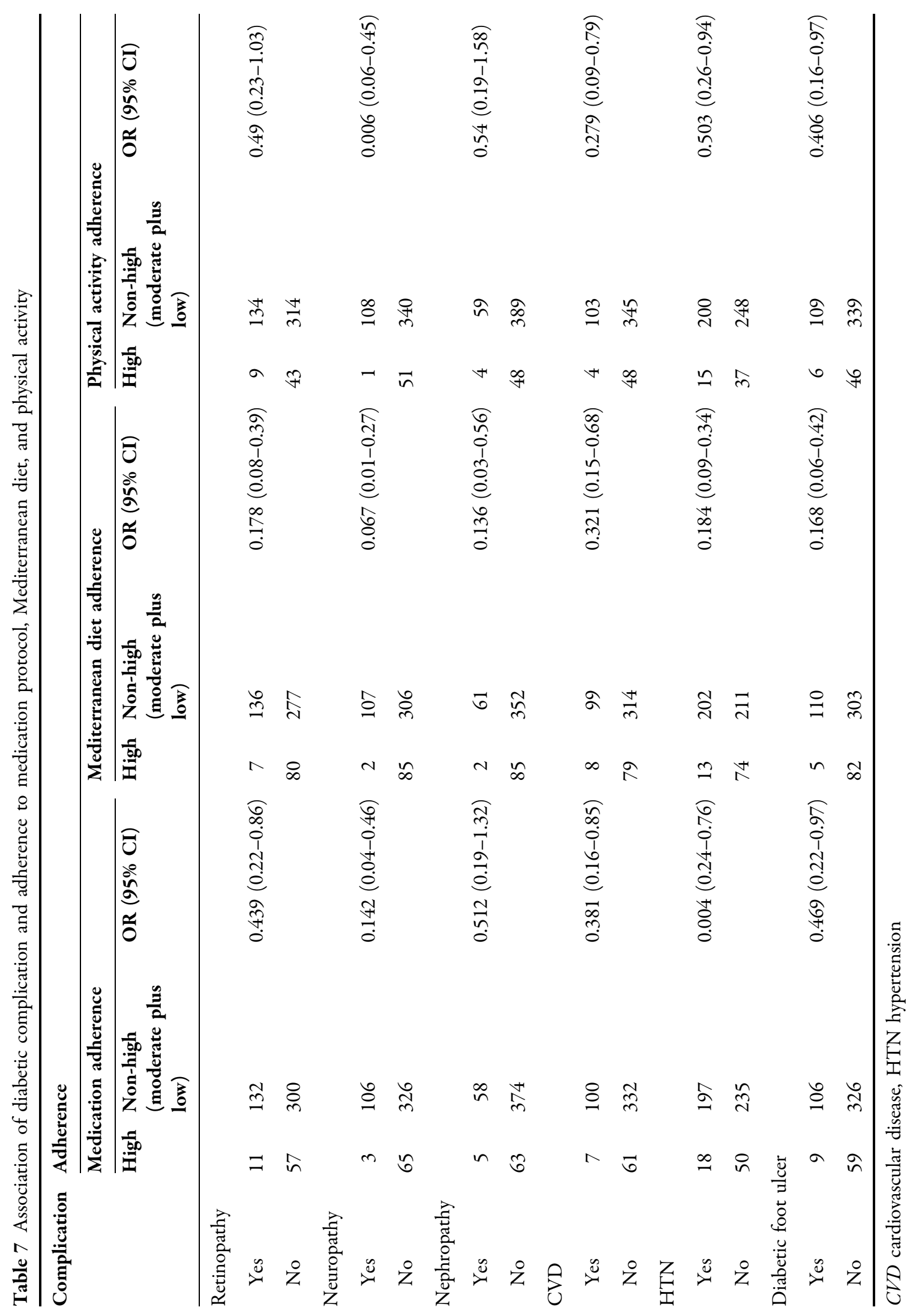


levels of low, moderate, and high adherence to medication protocol were $10.7 \%, 34.5 \%$, and $54.8 \%$, respectively [4]. Furthermore, increasing the level of adherence to medication related to lowering HbA1c was reasonable. Findings of the current study and those from the study by Doggrell and Warot confirm this relationship amongst patients with diabetes [26].

Adherence to medication can be improved by analyzing the associated factors. In the present study, there was a reduction in adherence to medication as the age of participants increased. This finding was in line with research in Malaysia, which showed that reduction of 1 year in the age of participants increased the probability of non-adherence to medication by $3.4 \%[27,28]$; however, another study did not find any relationship between age and adherence to medication [29]. These differences can be explained in part by the different socioeconomic status of the studied population. In addition, our findings revealed that people with normal weight had higher adherence to medication in comparison to overweight and obese individuals. This finding was in line with the SHIELD study [30]. It is notable that one of the potential confounding factors might be the high prevalence of depression amongst obese people, which could lead to decrease in medication adherence [28, 31]. Moreover, our findings indicated that the relationship between gender and adherence to medication was not significant. This important finding is consistent with the study by Tiv et al. $[29,32]$. Contrary to our expectations, we did not find a significant difference between educational level and adherence to medication. This result seem to be consistent with a recent study [29]. However, the findings of the current study did not support a significant relation between educational level and adherence to medication [33]. Finally, current smokers and ex-smokers had less adherence to medication compared to nonsmokers. This finding was also reported by Sherman and Lynch [34].

Dietary adherence of patients with diabetes is necessary for effective therapy and regular glycemic control. Several studies have shown that MD is a suitable diet in the nutritional management of diabetes. In the current study, the level of high adherence to MD was 17\% (85 patients) higher than in the previous studies amongst patients with CVD, which showed 2\% high adherence to MD [35]. This result was lower than that in research by Esposito et al., which indicated that $24 \%$ of patients with diabetes had high adherence to MD [6]. One unexpected finding was that $77.2 \%$ of patients had moderate adherence to MD. This finding was higher than the previous studies, which showed 55\% adherence to MD in CVD [35] and $50 \%$ in patients with diabetes [6]. A possible explanation for this could be increased awareness amongst patients with diabetes as a result of annual diabetic campaigns and patient education at the registered diabetic clinics.

In this research, while there was a positive relationship between adherence to MD and age groups (50-64 and at least 65 years of age), overweight, obesity, and smoker, there was no relationship between adherence to $\mathrm{MD}$ and gender, family history of diabetes, type of medication, level of education, marital status, and hookah smokers. On the basis of previous research, adherence to MD was inversely correlated with BMI amongst adolescents [36], and there was no significant relationship between adherence to MD and BMI in healthy adults [37]. This discrepancy could be attributed to differences in the characteristics of the study population. Furthermore, contrary to the current finding, a previous study by Parajuli et al. found that male participants adhered more to their MD than female ones did [38]. Although marital status had no significant association with adherence to MD in our research, Albuquerque et al.'s research found that being single had high a correlation with adherence to MD amongst patients with diabetes [39]. As well as contributing factors for non-compliance with diet, Parajuli et al.'s research included divorced status, negative family history of diabetes mellitus, and low knowledge of diabetes mellitus [38]. On the basis of previous research, adherence to MD can be related to PA and educational level [14]. This discrepancy could be attributed to different sociocultural differences.

Regular PA is a primary component in management of T2D. Data from a review of the literature revealed a link between regular PA and 
glycemic control amongst patients with diabetes [12]. Low adherence to PA is a result of sedentary lifestyle, which increased the risk factors for cardiometabolic diseases [40]. In the current study, low adherence to PA was observed in $21.2 \%$ (105 patients), but in previous studies in the normal and diabetic population the corresponding value was $40 \%$ (in the total Iranian population) and 45\% (in the diabetic population), respectively [41]. One possible explanation for this could be the growing awareness of patients with diabetes that has contributed to reducing the frequency of sedentary lifestyle in the last decade. Another aspect in the present research was moderate PA of $25.6 \%$, which was greater than moderate adherence to PA of $43 \%$ in another study [42].

In the current study, there was a significant relationship between smoking status and adherence to PA, but there was no relationship between adherence to PA and gender, age group, BMI, marital status, type of medication, having a glucometer, and hookah smoker. However, factors for low adherence to PA in previous study were divorce status, negative family history of diabetes mellitus, and low socioeconomic status [38]. To sum up, as a result of the diversity in factors contributing adherence to PA and sedentary lifestyle amongst the study population, sociodemographic approaches to interpreting PA might be warranted [43, 44].

Adherence to medication, MD, and physical activity amongst patients with T2D can have an influence on complications and comorbidities. Our findings indicate that adherence to medication protocol reduces the chance of retinopathy, nephropathy, neuropathy, CVD, HTN, and diabetic foot ulcer. However, caution must be applied and future studies are essential when interpreting the results. Overall, our findings indicate that adherence to MD can reduce the chance of retinopathy, nephropathy, neuropathy, CVD, HTN, and diabetic foot ulcer. Moreover, our findings indicate that adherence to PA reduces the chance of retinopathy, nephropathy, neuropathy, CVD, HTN, and diabetic foot ulcer [2].

A limitation of study was the unavailability of more than 4 years' worth of documentation because these special diabetes clinics only started in Iran in recent years. Another limitation could be the small sample size used for further analysis.

\section{CONCLUSION}

Even though the prevalence of diabetic and special diabetic clinics has increased, levels of adherence to medications, diet, and PA amongst patients with T2D are not within an acceptable range. Overall, the current findings showed that reduced levels of adherence to medication protocol, MD, and PA were associated with increased HbA1c. Also, failure to achieve optimal control might be linked to insufficient understanding of the associated factors. Despite the wide variety of factors contributing to adherence to medications, diet, and PA among patients with T2D, adherence-associated factors could be added to the diabetic guidelines to improve glycemic management. In addition, on the basis of the associated factors, an educational package for healthcare providers in primary care centers should be developed and tailored to convey an appropriate message for promoting adherence to medication, diet, and physical activity among patients with T2D.

\section{ACKNOWLEDGEMENTS}

All data generated or analyzed during this study are included in this published article. We thank the participants of the study.

Funding. This article is the result of a Master's Thesis supported by Shiraz University of Medical Sciences (18110). No Rapid Service Fee was received by the journal for the publication of this article.

Editorial Assistance. The authors wish to thank Mr. H. Argasi of the Research Consultation Center (RCC) of Shiraz University of Medical Sciences for his invaluable assistance in editing this manuscript. This assistance was funded by Shiraz University of Medical Sciences. 
Authorship. All named authors meet the International Committee of Medical Journal Editors (ICMJE) criteria for authorship for this article, take responsibility for the integrity of the work as a whole, and have given their approval for this version to be published.

Authorship Contributions. HK and MHS contributed to study conception and design. AM contributed to the study design, HK data acquisition and data analysis. MS contributed to the study design and statistical analysis. All authors interpreted the data and reviewed and edited the manuscript for important intellectual content. All authors have read and approved the final version of the manuscript.

Disclosures. Alireza Mirahmadizadeh, Haniye Khorshidsavar, Mozhgan Seif, and Mohammad Hossein Sharifi have nothing to disclose.

Compliance with Ethics Guidelines. This study was approved by the local Ethics Committee of Shiraz University of Medical Sciences (SUMS); IR.SUMS.REC.1397.972. In addition, written informed consent was obtained from each participant. This study was performed in accordance with the Helsinki Declaration of 1964 and its later amendments.

Open Access. This article is distributed under the terms of the Creative Commons Attribution-NonCommercial 4.0 International License (http://creativecommons.org/licenses/ by-nc/4.0/), which permits any noncommercial use, distribution, and reproduction in any medium, provided you give appropriate credit to the original author(s) and the source, provide a link to the Creative Commons license, and indicate if changes were made.

\section{REFERENCES}

1. Zheng Y, Ley SH, Hu FB. Global aetiology and epidemiology of type 2 diabetes mellitus and its complications. Nat Rev Endocrinol. 2018;14(2):88.

2. Lerman I. Adherence to treatment: the key for avoiding long-term complications of diabetes. Arch Med Res. 2005;36(3):300-6.
3. Cho N, Shaw J, Karuranga S, et al. IDF Diabetes Atlas: global estimates of diabetes prevalence for 2017 and projections for 2045. Diabetes Res Clin Pract. 2018;138:271-81.

4. Ahmed NO, Abugalambo S, Almethen GH. Adherence to oral hypoglycemic medication among patients with diabetes in Saudi Arabia. Int J Health Sci. 2017;11(3):45.

5. Elsous A, Radwan M, Al-Sharif H, Abu Mustafa A. Medications adherence and associated factors among patients with type 2 diabetes mellitus in the Gaza Strip, Palestine. Front Endocrinol. 2017;8:100.

6. Esposito K, Maiorino M, Di Palo C, Giugliano D, Campanian Postprandial Hyperglycemia Study Group. Adherence to a Mediterranean diet and glycaemic control in type 2 diabetes mellitus. Diabet Med. 2009;26(9):900-7.

7. Schwingshackl L, Missbach B, König J, Hoffmann G. Adherence to a Mediterranean diet and risk of diabetes: a systematic review and meta-analysis. Public Health Nutr. 2015;18(7):1292-9.

8. Tobias Ferrer J, Santasusana Riera I, Cuadrench Solórzano M, Gonzalez Cabré M, Girbau Tapias M, Sant Masoliver C. Adherence to the Mediterranean diet in patients with coronary artery disease. Rev Esp Cardiol (Engl Ed). 2015;68(1):73-5.

9. Esposito K, Maiorino MI, Ciotola M, et al. Effects of a Mediterranean-style diet on the need for antihyperglycemic drug therapy in patients with newly diagnosed type 2 diabetes: a randomized trial. Obstet Gynecol Surv. 2010;65(6):379-80.

10. Forechi L, Mill JG, Griep RH, Santos I, Pitanga F, Molina MDCB. Adherence to physical activity in adults with chronic diseases: ELSA-Brasil. Rev Saude Publica. 2018;52:31.

11. Saint-Maurice PF, Troiano RP, Matthews CE, Kraus WE. Moderate-to-vigorous physical activity and allcause mortality: do bouts matter? J Am Heart Assoc. 2018;7(6):e007678.

12. Sigal RJ, Armstrong MJ, Bacon SL, et al. Physical activity and diabetes. Can J Diabetes. 2018;42: S54-63.

13. Bullard T, Ji M, An R, Trinh L, Mackenzie M, Mullen SP. A systematic review and meta-analysis of adherence to physical activity interventions among three chronic conditions: cancer, cardiovascular disease, and diabetes. BMC Public Health. 2019;19(1):636.

14. Qiu S-H, Sun Z-L, Cai X, Liu L, Yang B. Improving patients' adherence to physical activity in diabetes 
mellitus: a review. Diabetes Metab J. 2012;36(1): $1-5$.

15. Bommer C, Sagalova V, Heesemann E, et al. Global economic burden of diabetes in adults: projections from 2015 to 2030. Diabetes Care. 2018;41(5): 963-70.

16. Cohen J. Statistical power analysis for the behavioral sciences. Abingdon: Routledge; 2013.

17. Montgomery DC. Design and analysis of experiments. New York: Wiley; 2017.

18. Rodríguez-Ortiz D, Reyes-Pérez A, León P, et al. Assessment of two different diagnostic guidelines criteria (National Cholesterol Education Adult Treatment Panel III [ATP III] and International Diabetes Federation [IDF]) for the evaluation of metabolic syndrome remission in a longitudinal cohort of patients undergoing Roux-en-Y gastric bypass. Surgery. 2016;159(4):1121-8.

19. Ghanei Gheshlagh R, Ebadi A, Veisi Raygani A, Nourozi Tabrizi K, Dalvandi A, Mahmoodi H. Determining concurrent validity of the Morisky medication adherence scale in patients with type 2 diabetes. Iran J Rehabil Res Nurs. 2015;1(3):24-32.

20. Gholamaliei B, Karimi-Shahanjarini A, Roshanaei G. Medication adherence and its related factors in patients with type II diabetes. J Educ Community Health. 2016;2(4):3-12.

21. Committee IR. Guidelines for data processing and analysis of the International Physical Activity Questionnaire (IPAQ)—short and long forms. 2005. http://www.ipaqkise/scoring.pdf.

22. Moghaddam MB, Aghdam FB, Jafarabadi MA, Allahverdipour H, Nikookheslat SD, Safarpour S. The Iranian version of International Physical Activity Questionnaire (IPAQ) in Iran: content and construct validity, factor structure, internal consistency and stability. World Appl Sci J. 2012;18(8): 1073-80.

23. Ainsworth BE, Haskell WL, Herrmann SD, et al. 2011 compendium of physical activities: a second update of codes and MET values. Med Sci Sports Exerc. 2011;43(8):1575-81.

24. Committee IR. Guidelines for data processing and analysis of the International Physical Activity Questionnaire (IPAQ)-short and long forms. http:// www.ipaqkise/scoring.pdf. 2005.

25. Polonsky WH, Henry RR. Poor medication adherence in type 2 diabetes: recognizing the scope of the problem and its key contributors. Patient Prefer Adherence. 2016;10:1299.
26. Doggrell SA, Warot S. The association between the measurement of adherence to anti-diabetes medicine and the HbA1c. Int J Clin Pharm. 2014;36(3): 488-97.

27. Ahmad NS, Ramli A, Islahudin F, Paraidathathu T. Medication adherence in patients with type 2 diabetes mellitus treated at primary health clinics in Malaysia. Patient Prefer Adherence. 2013;7:525.

28. Chew B-H, Hassan N-H, Sherina M-S. Determinants of medication adherence among adults with type 2 diabetes mellitus in three Malaysian public health clinics: a cross-sectional study. Patient Prefer Adherence. 2015;9:639.

29. Waari G, Mutai J, Gikunju J. Medication adherence and factors associated with poor adherence among type 2 diabetes mellitus patients on follow-up at Kenyatta National Hospital, Kenya. Pan Afr Med J. 2018;29(1):1-15.

30. Grandy S, Fox KM, Hardy E, SHIELD Study Group. Association of weight loss and medication adherence among adults with type 2 diabetes mellitus: SHIELD (Study to Help Improve Early evaluation and management of risk factors Leading to Diabetes). Curr Ther Res. 2013;75:77-82.

31. Quek YH, Tam WW, Zhang MW, Ho RC. Exploring the association between childhood and adolescent obesity and depression: a meta-analysis. Obes Rev. 2017;18(7):742-54.

32. Tiv M, Viel J-F, Mauny F, et al. Medication adherence in type 2 diabetes: the ENTRED study 2007, a French population-based study. PLoS One. 2012;7(3):e32412.

33. Mashrouteh M, Khanjani N, Gozashti MH. Evaluation of compliance with drug regimens in diabetic patients referred to the endocrinology clinic of Afzalipour Hospital, Kerman, Iran. J Health Dev. 2012;1(3):182-92.

34. Sherman BW, Lynch WD. The association of smoking with medical treatment adherence in the workforce of a large employer. Patient Prefer Adherence. 2014;8:477.

35. Mahdavi-Roshan M, Salari A, Gholipour M, Naghshbandi M. Dietary adherence in people with cardiovascular risk factors living in northern Iran. J Babol Univ Med Sci. 2017;19(1910):62-8.

36. Martin-Calvo N, Chavarro JE, Falbe J, Hu FB, Field AE. Adherence to the Mediterranean dietary pattern and BMI change among US adolescents. Int J Obes (Lond). 2016;40(7):1103.

37. Inan-Eroglu E, Çetin C, Mutlu AA, Ayaz A, Samur G. Does adherence to Mediterranean diet affect body 
mass index and dietary macronutrient intakes in adults? Clin Nutr. 2018;37:S112.

38. Parajuli J, Saleh F, Thapa N, Ali L. Factors associated with nonadherence to diet and physical activity among Nepalese type 2 diabetes patients; a cross sectional study. BMC Res Notes. 2014;7(1):758.

39. Albuquerque C, Correia C, Ferreira M. Adherence to the therapeutic regime in person with type 2 diabetes. Procedia-Social and Behavioral Sciences. 2015;171:350-8.

40. Aune D, Schlesinger S, Norat T, Riboli E. Diabetes mellitus and the risk of sudden cardiac death: a systematic review and meta-analysis of prospective studies. Nutr Metab Cardiovasc Dis. 2018;28(6): 543-56.

41. Sahebkar M, Heidarian Miri H, Noormohammadpour $\mathrm{P}$, et al. Prevalence and correlates of low physical activity in the Iranian population: national survey on non-communicable diseases in 2011. Scand J Med Sci Sports. 2018;28(8):1916-24.

42. Dashtian M, Eftekhar Ardebili H, Karimzadeh Shirazi K, Shahmoradi M, Azam K. Predicting factors affecting medication adherence and physical activity in patients with type- 2 diabetes mellitus based on the theory of planned behavior. J School Public Health Inst Public Health Res. 2017;15(2): 133-46.

43. Bergman P, Grjibovski AM, Hagströmer M, Bauman A, Sjöström M. Adherence to physical activity recommendations and the influence of socio-demographic correlates-a population-based crosssectional study. BMC Public Health. 2008;8(1):367.

44. Alkerwi A, Schuh B, Sauvageot N, et al. Adherence to physical activity recommendations and its associated factors: an interregional population-based study. J Public Health Res. 2015;4(1):406. 